\title{
The Relevance of Salutogenesis to Social Issues Besides Health: The Case of Sense of Coherence and Intergroup Relations
}

\author{
Shifra Sagy and Adi Mana
}

\section{Introduction}

Antonovsky's salutogenic model and its core concept "sense of coherence" (SOC) focus on the ability of individuals to cope with stressors in life and stay healthy. Accordingly, the relationship between SOC and health has received much attention and quite consistent results in research (see Part III of this book). However, since the paradigm of salutogenesis was suggested in the discipline of sociology of health, the relationships between salutogenesis and other social concepts have been mostly neglected. Unfortunately, up to now, the salutogenic model has never been broadened into an interdisciplinary framework. We believe that at the dawn of our new millennium, which poses new challenges of interdisciplinarity in research and academic studies (Gruenwald, 2014), the salutogenic paradigm should broaden its scope. When we propose broadening salutogenesis into interdisciplinarity, we may consider disciplines such as psychology, economics, geography, and anthropology. Thus, this chapter aims to raise some new questions in the framework of the salutogenic paradigm toward interdisciplinarity and to review the few studies which have already attempted to deal with it.

First, we must deal with the core dilemma: Does the salutogenic orientation enable us to deal with other concepts beyond the relationship with health? And if so, how? How can we ask salutogenic questions employing concepts embedded in other disciplines?

Let us start with the first question. In his writing about the development of SOC, Antonovsky wrote extensively about

\footnotetext{
S. Sagy $(\bowtie)$

Department of Education, Ben-Gurion University of the Negev,

Beersheba, Israel

e-mail: shifra@bgu.ac.il

A. Mana

School of Behavioral Sciences, Peres Academic Center, Rehovot, Israel

e-mail: manna.adi@gmail.com
}

how one's life situation can influence his/her strength of SOC (Antonovsky, 1987). In his dealing with this special issuethe origins of SOC-he expressed an interdisciplinary approach by relating to broad ranging factors like culture, social forces, social position, gender, ethnicity, genetics, or even plain luck (Benz, Bull, Mittelmark, \& Vaandrager, 2014). Indeed, social factors have seldom been studied as predictors of SOC (e.g., Sagy \& Antonovsky, 2000; Lam, 2007 and others). Two aspects in particular have mainly been studied: the experience of cultural integration vs. discrimination due to being part of a minority group (Ying et al., 2001), and the experience of cultural stability vs. instability (e.g., Antonovsky \& Sagy, 1986).

Even though this body of research was mostly composed of correlational studies, which leave the direction of causality undetermined, in most of the studies, the suggested direction was that elements related to a variety of social factors should be considered as predictors of SOC.

However, while Antonovsky discussed the other direction of the equation, meaning how SOC can influence life situations, his answer was much less interdisciplinary. As Antonovsky's PhD student (the first author) I heard answers which seemed to be completely clear: "A salutogenic orientation, no less than a pathologic one, defines health and disease only in terms of functioning and survival. All that it argues is that the stronger the SOC, the more likely the system, whether individual, family or society, to function and survive" (Antonovsky, 1991, p. 8). What he meant was that SOC, whose development is influenced by social factors, cannot predict social concepts, which could have positive or negative connotations. Moreover, Antonovsky viewed such social factors as loaded by moral-philosophical problems, and as such, not inherently within the context of salutogenesis (Antonovsky, 1991). This original conviction of his can partly explain the focus of salutogenic research over almost four decades on the SOC/health hypothesis and why it has not been broadened to include other interdisciplinary concepts as well. 
By raising this question again, we assume that salutogenesis, 40 years later, is challenged by the call of interdisciplinarity. We believe that researchers can ask salutogenic questions in different areas, not only health and well-being, and perhaps find other salutogenic answers. When we employ an interdisciplinary approach, the salutogenic question then would not only be "Who copes successfully and stays healthy?" (Antonovsky \& Sagy, 1986) but, for example, "Who expresses more openness to the "other"?" or "Who is a social activist who pursues justness in the world"? Or "Who is a peacemaker?" If we broaden the level, we can ask about salutogenic schools, neighborhoods, or other social institutions which enhance not only health and wellness but also justice, peace, and reconciliation. The definitions of these concepts can vary over different contexts and cultures, but the question stays a salutogenic one.

Now we arrive at the second part of our review: How can we ask salutogenic questions in an interdisciplinary framework? Relating to this "how" question, we review some studies which have already attempted to do so. Our interdisciplinary review starts with some studies which found positive interactions between SOC and the relations between members of the same community (e.g., Maass, Lindström, \& Lillefjell, 2014; Morton \& Lurie, 2013; Teig et al., 2009). Indeed, this evidence can be explained by the well-known relationship between strong social connection or connectivity and enhanced sense of health and well-being (Vaandrager $\&$ Kennedy, 2016). This explanation brings us back to the SOC/health equation. However, other explanations, embedded in social psychology theories, can be considered as well.

A second area of research deals with the relationship between SOC and social relations with out-group members. A small qualitative study (Griffiths, Ryan, \& Foster, 2011) suggests an interesting explanation for the small amount of research on SOC and intergroup relations. The researchers used qualitative research methodology to explore how SOC was applied in daily life. Their findings suggest that SOC may be split into coping and adaptive capacities for concrete problems vis-a-vis social relations. Thus, a strong level of SOC was found to be effective in dealing with nonrelationship oriented problems but less effective in dealing with social relations oriented problems. It appears that different sets of resources, but not SOC, are required for dealing with the latter.

Other studies have attempted to explore the relationship between SOC and personal traits which could facilitate social relations with out-group members. Feldt, Metsäpelto, Kinnunen, and Pulkkinen (2007) analyzed the relations between SOC and the five-factor model of personality. The results indicate that a person with a strong SOC shows modest positive associations with extraversion, openness, conscientiousness, and agreeableness. Another study
(Pålsson et al., 1996) found negative correlations between SOC and personality traits of avoidance, detachment, hostility, and aggression and positive correlations between SOC and empathy.

In the political psychology area, SOC was measured as a predictor of different political attitudes. No correlations were found between SOC and attitude scales measuring patriotism, nationalism, and authoritarianism (Renner, Salem, \& Alexandrowicz, 2004). However, political attitudes toward peace were found in correlation with strong SOC (Braun-Lewensohn, Abu-Kaf, \& Sagy, 2015). In a study of Israeli adolescents during a politically violent event, we found a link between SOC and belief in peaceful ways of resolution in the context of the Israeli-Palestinian conflict. It appears that adolescents who had a strong SOC, also had a strong tendency to view the conflict as another challenge in life, perceived the conflict as manageable and as meaningful to cope with. This study, however, was a one-time, cross-sectional study and its causal interpretation could be also different.

In sum, the findings related to the relationship between SOC and personal traits or attitudes which facilitate social relations are quite ambiguous. However, other studies should be carried out to support their promising results. The prominent directions revealed from this body of research is that a strong SOC is connected with tendencies associated with positive values, at least in Western society. Are these conclusions that Antonovsky tried to avoid? Perhaps yes. In his lecture in Prague (Antonovsky, 1991) he indeed warned of the danger of defining health so that it becomes ". . a catchall for anything that you think is good. Health then becomes not a scientific concept, but confused with a set of answers to moral-philosophical problems. . .the distinction must be made" (Antonovsky, 1991, p. 9). We deeply understand these warnings and accept them. In a later article (Antonovsky, 1995, p. 11), he warned against the danger of assuming that "the morally good is salutary." However, when we broaden salutogenesis to include concepts other than physical health, we cannot avoid these moral-philosophical questions about values and science. Thus, we have to deal with them while fully recognizing our limitations in making such a distinction.

Another direction of research which seems meaningful to be included in this review relates to larger social systems than the individual. Since attitudes and behaviors toward the out-group are developed within one's social context, the relationship between SOC and intergroup relations should be explored not only from the individual perspective but also in the supra-system context (Sagy \& Sarid, 2015). The idea that the SOC concept should be broadened to larger levels than individuals has been suggested and discussed by Sagy and Antonovsky (Sagy, 1990; Antonovsky, 1992; Sagy \& Antonovsky, 1992). It is beyond the scope of this paper to 
include this extensive discussion. In this chapter, we review some studies that link the SOC of the collective to intergroup and social relations.

The concept of community sense of coherence (CSOC) was developed as related to a specific in-group and not to the "global orientation of the world" as it is defined for individual SOC. It constitutes the three components of SOC: comprehensibility, manageability, and meaningfulness (BraunLewensohn \& Sagy, 2011; Elfassi, Braun-Lewensohn, Krumer-Nevo, \& Sagy, 2016; Peled, Sagy, \& BraunLewensohn, 2013; Sagy, 1998). Community comprehensibility relates to the perception that life in one's community is predictable, safe, and secure and that one's community is a place which is known and understood. Community manageability relates to the perception that one's community can assist its members, is available to them, and meets their demands and needs. Lastly, community meaningfulness relates to perception that the community gives meaning to its members, provides challenges, and is worthy of investment and engagement.

Indeed, most of the research which investigated the concept of CSOC has focused on its relationship with well-being and resilience. Just recently, however, a few studies have attempted to connect salutogenesis and SOC to other social concepts such as intergroup relations, openness toward the "other" and readiness to reconcile (Mana, Sagy, \& Srour, 2015; Sagy, 2014; Srour, 2015). These studies connect the salutogenic paradigm with other interdisciplinary models and concepts such as social identity (Tajfel, 1981), acculturation (Berry, 1990), conflict studies (Bar-Tal, 1998), or peace and reconciliation (Nadler, 2012).

Most of these new studies examined the relationship between CSOC and intergroup relations. The relations between the conflicted groups were examined by the levels of adherence to in-group as well as acceptance of the out-group collective narratives and acculturation attitudes. One of the studies was conducted among Palestinian Muslims and Christians in Israel (Mana et al., 2015). The results revealed that strong community sense of coherence (CSOC) was correlated with higher levels of acceptance of the in-group collective narrative and with lower levels of acceptance of the out-group collective narratives. Community sense of coherence was also related to higher levels of a tendency to adopt a separation strategy between the two groups in conflict. The authors based their explanation on a wide range of studies in social psychology which suggest that group members, who believe that their own group and its products are superior to other groups, are prone to behaviorally discriminate against other groups (e.g., Bizumic \& Duckitt, 2009). This notion was well established in the work of Tajfel (1981) who analyzed three cognitive aspects of prejudice: the process of categorization, which gives shape to intergroup attitudes, the process of assimilation of social values and norms which provides their content, and sense of coherence, as a main cognitive aspect which relates to the way individuals react to specific intergroup situations. Following Tajfel's paradigm, a strong CSOC enables group members to deal with changes that occur in intergroup situations. In order to deal with these changes, an individual must make constant attributions that help him deal with the new situations in a manner that appears consistent to him and preserves his self-image and integrity. The individual needs to build a cognitive structure which provides him with a satisfactory explanation of the causes of changes. However, an improved group position is often achieved by using the group's capacity to put another group at a disadvantage and derives largely from biased comparisons on salient dimensions that are favorable to the in-group and unfavorable to the out-group (Tajfel, 1981).

A wider concept recently suggested by Sagy (2014) relates to national sense of coherence (NSOC). Two studies are presently being conducted among Israeli-Jews and Israeli-Arabs. The initial results indicate a strong negative correlation between strong NSOC and level of openness to the narrative of the "other" group. Strong NSOC was also found as negatively related to readiness to reconcile with the “other" among Israeli Jewish students (Sagy \& Sarid, 2015).

These studies pose different salutogenic questions which do not concern SOC/health but SOC/social relations. The interdisciplinary salutogenic questions in these studies are: How does a collective with a strong SOC perceive, feel, or behave toward the "other"? Is the tendency of a group to perceive its world as comprehensible, meaningful, and manageable related to greater openness to the "other," or does it involve clinging to the rigid in-group identity and less openness toward the "other"? Is an individual, a group, a collective, or a system with a stronger SOC more likely to live in peace/justice/good relations with their surroundings?

To sum up, while there is a broad consensus regarding the contribution of SOC to health and well-being, the role of SOC - both of the individual or the collective-in social relations has been mostly neglected. We maintain that one of the reasons for this neglect lies in the initial excitement of Antonovsky and his followers about study the SOC/health connection. Times have changed and interdisciplinarity seems to be the challenge of our era. In this chapter, we have attempted to review the small body of research which asks other salutogenic questions relating SOC to other interdisciplinary concepts.

We believe that more research is needed in order to gain a deeper understanding of these initial answers. Moreover, interdisciplinarity can also lead to employing other salutogenic concepts-rather than SOC-to give answers to salutogenic questions. So we suggest that salutogenic researchers in the future not only ask new salutogenic questions but also develop new salutogenic concepts in the 
attempt to broaden and deepen our understanding of the paradigm. We hope that this chapter succeeds in posing this new challenge.

Open Access This chapter is distributed under the terms of the Creative Commons Attribution-Noncommercial 2.5 License (http:// creativecommons.org/licenses/by-nc/2.5/) which permits any noncommercial use, distribution, and reproduction in any medium, provided the original author(s) and source are credited.

The images or other third party material in this chapter are included in the work's Creative Commons license, unless indicated otherwise in the credit line; if such material is not included in the work's Creative Commons license and the respective action is not permitted by statutory regulation, users will need to obtain permission from the license holder to duplicate, adapt or reproduce the material.

\section{References}

Antonovsky, A. (1987). Unraveling the mystery of health. San Francisco: Jossey-Bass.

Antonovsky, A. (1991). The salutogenic approach to family system health: Promise and danger. Lecture at European Congress on "Mental Health in European Families", Prague, Czechoslovakia, 5-8 May 1991. Retrieved from http://www.angelfire.com/ok/soc/ agolem.html.

Antonovsky, A. (1992). Can attitudes contribute to health? Advances, 8 , 33-49.

Antonovsky, A. (1995). The moral and the healthy: Identical, overlapping, or orthogonal? Israel Journal of Psychiatry and Related Sciences, 32(1), 5-13.

Antonovsky, H., \& Sagy, S. (1986). The development of a sense of coherence and its impact on responses to stress situations. Journal of Social Psychology, 126, 213-227.

Bar-Tal, D. (1998). Social beliefs in times of intractable conflict: The Israeli case. International Journal of Conflict Management, 9, 22-50.

Benz, C., Bull, T., Mittelmark, M., \& Vaandrager, L. (2014). Culture in salutogenesis: The scholarship of Aaron Antonovsky. Global Health Promotion, 21(4), 16-23.

Berry, J. W. (1990). Psychology of acculturation. In J. J. Berman (Ed.), Nebraska Symposium on Motivation, 1989: Cross-cultural perspectives. Current theory and research in motivation (Vol. 37, pp. 201-235). Lincoln, NE: University of Nebraska Press.

Bizumic, B., \& Duckitt, J. (2009). Narcissism and ethnocentrism: A review. Directions in Psychiatry, 29, 99-109.

Braun-Lewensohn, O., Abu-Kaf, S., \& Sagy, S. (2015). Attitudes toward war and peace and their relations with anxiety reactions among adolescents living in a conflictual area. Journal of Youth Studies, 18(1), 68-79.

Braun-Lewensohn, O., \& Sagy, S. (2011). Salutogenesis and culture. Personal and community sense of coherence among adolescents belonging to three different cultural groups. International Review of Psychiatry, 23(6), 533-541.

Elfassi, Y., Braun-Lewensohn, O., Krumer-Nevo, M., \& Sagy, S. (2016). Community sense of coherence among adolescents as related to their involvement in risk behaviors. Journal of Community Psychology, 44(1), 22-37.

Feldt, T., Metsäpelto, R.-L., Kinnunen, U., \& Pulkkinen, L. (2007). Sense of coherence and five-factor approach to personality: Conceptual relationships. European Psychologist, 12(3), 165-172.

Griffiths, C. A., Ryan, P., \& Foster, J. H. (2011). Thematic analysis of Antonovsky's sense of coherence theory. Scandinavian Journal of Psychology, 52(2), 168-173.
Gruenwald, O. (2014). The promise of interdisciplinary studies: Re-imaging the university. Journal of Interdisciplinary Studies, $26,1-28$.

Lam, B. T. (2007). Impact of perceived racial discrimination and collective self-esteem on psychological distress among Vietnamese-American college students: Sense of coherence as mediator. American Journal of Orthopsychiatry, 77(3), 370-376.

Maass, R., Lindström, B., \& Lillefjell, M. (2014). Exploring the relationship between perceptions of neighborhood-resources, sense of coherence and health for different groups in a Norwegian neighbourhood. Journal of Public Health Research, 3(208).

Mana, A., Sagy, S., \& Srour, A. (2015). Sense of community coherence and inter-religious relations. Journal of Social Psychology. doi:10. 1080/00224545.2015.1129302.

Morton, M. J., \& Lurie, N. (2013). Community resilience and public health practice. American Journal of Public Health, 103(7), $1158-1160$.

Nadler, A. (2012). Intergroup reconciliation: Definition, processes, and dilemmas. In L. Tropp (Ed.), Oxford handbook of conflict. New York: Oxford University Press.

Pålsson, M. B., Hallberg, I. R., Norberg, A., \& Björvell, H. (1996). Burnout, empathy, and sense of coherence among Swedish district nurses before and after systematic clinical supervision. Scandinavian Journal of Caring Sciences, 10(1), 19-26.

Peled, D., Sagy, S., \& Braun-Lewensohn, O. (2013). Community perceptions as coping resource among adolescents living under rocket fire: A salutogenic approach. Journal of Community Positive Practices, 4, 681-702.

Renner, W., Salem, I., \& Alexandrowicz, R. (2004). Human values as predictors for political, religious, and health-related attitudes: A contribution towards validating the Australian Value Questionnaire (AVQ) by structural equation modeling. Social Behavior and Personality, 32(5), 477-490.

Sagy, S. (1990). The family sense of coherence and adjustment to stressors. Unpublished doctoral dissertation. Ben-Gurion University of the Negev, Beersheva, Israel (Hebrew).

Sagy, S. (1998). Effects of personal, family, and community characteristics on emotional reactions in a community stress situation: The Golan Heights negotiations. Youth \& Society, 29(3), 311-329.

Sagy, S. (2014). Salutogenesis: Notes from the diary of a conflict researcher in the "safe room" during "Operation Pillar of Cloud.". Mifgash: Journal of Social-Education Work, 40, 9-26. Hebrew.

Sagy, S., \& Antonovsky, A. (1992). The family sense of coherence and the retirement transition. Journal of Marriage and the Family, 54, 983-993.

Sagy, S., \& Antonovsky, H. (2000). The development of the Sense of Coherence: A retrospective study of early life experiences in the family. International Journal of Aging \& Human Development, 51, 155-166.

Sagy, S., \& Sarid, A. (2015). Does our national sense of coherence influence our willingness to accept the "other": Before and after the war in Gaza." Presented at the Conference of Democracy and Racism. Tel Aviv, Israel.

Srour, A. (2015). Community sense of coherence, collective narrative perceptions and openness to the "other" group: The case of Muslims-Christians relations in Israel. Ph.D. thesis. Ben Gurion University (Hebrew).

Tajfel, H. (1981). Human groups and social categories: Studies in social psychology. Cambridge: Cambridge University Press.

Teig, E., Amulya, J., Bardwell, L., Buchenau, M., Marshall, J. A., \& Litt, J. S. (2009). Collective efficacy in Denver, Colorado: Strengthening neighborhoods and health through community gardens. Health \& Place, 15(4), 1115-1122. doi:10.1016/j. healthplace.2009.06.003. 
Telaku, M., \& Sagy, S. (forthcoming). Perceptions of collective narratives and acculturation attitudes: The case of Albanians and Serbs in Kosovo. In L. Gardner-Feldman, R. Barash, \& S. Goda (Eds.), Societies in Transition. The Former Soviet Union and East Central Europe between conflict and reconciliation. Vandenhoeck \& Ruprecht. The Jena Center for Reconciliation Studies' (JCRS) series on Research in Peace and Reconciliation (RIPAR).
Vaandrager, L., \& Kennedy, L. (2016). Communities and neighborhoods. In Handbook of Salutogenesis. New York: Springer. Chapter 14.

Ying, Y. W., Lee, P. A., Tsai, J. L., Hung, Y., Lin, M., \& Wan, C. T. (2001). Asian American college students as model minorities: An examination of their overall competence. Cultural Diversity \& Ethnic Minority Psychology, 7(1), 59-74. 